\title{
Modeling SARS-CoV-2 infection in mice
}

Sun, S-H. et al. Cell Host Microbe https://doi.org/10.1016/j.chom.2020.05.020 (2020)

Research efforts are in full swing to identify effective medical countermeasures against COVID-19. As with previous outbreaks, animal models will be instrumental for understanding SARS-CoV-2 pathogenesis, screening drugs and developing vaccines. Early studies have revealed that only a handful of animal models are susceptible to the new coronavirus, and most of them display only mild symptoms after infection. Macaques, closely related to humans, might predict better than other models how drugs and vaccines will work in humans, but few animal facilities can perform nonhuman primate experiments in high containment. Therefore, small animal models that reproduce the clinical signs and pathology observed in COVID-19 patients are highly needed.

SARS coronaviruses are inefficient at infecting mice due to structural differences between mouse angiotensin-converting enzyme 2 (ACE2) and human ACE2, the receptor facilitating virus entry and replication in humans. As a result, laboratories are using different strategies to generate mice that express human ACE2, to make them more susceptible to SARS-CoV-2 and more suitable for COVID-19 research.

In a new study published in Cell Host \& Microbe, investigators from the Academy of Military Medical Sciences and National Institutes for Food and Drug Control in Beijing generated humanized ACE2 mice (hACE2 mice) using a CRISPR/Cas9 knock-in technology. The targeting strategy was designed to insert human ACE2 cDNA into the first coding exon of the mouse Ace 2 gene, and place human ACE2 under the control of the endogenous mouse Ace 2 promoter.

To characterize the susceptibility of hACE2 mice to SARS-CoV-2 infection, young (4.5-week-old) and old (30-week-old) female hACE2 and wild-type (WT) C57BL/6 mice were intranasally infected with the virus. Infected mice did not develop clinical symptoms, but aged hACE2 mice lost weight. Viral RNA replication was detected by RT-qPCR in the lung, trachea, and brain tissues of both young and aged hACE2

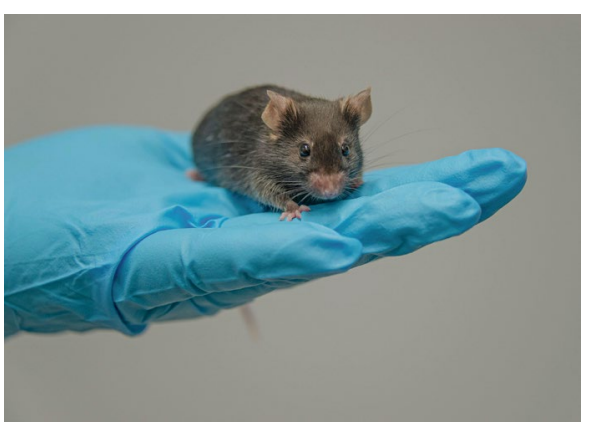

Credit: Oleg Senkov / Alamy Stock Photo.

mice, but not in WT mice. Viral S protein was detected by immunostaining along the airway in both young and aged hACE2 mice, predominantly in Clara cells positive for uteroglobulin, indicating that this cell type is a major target of the virus in this model. Histopathological analysis of the lungs showed that both young and aged hACE2 mice developed interstitial pneumonia with inflammatory cell infiltration. Lesions were more severe in aged hACE2 mice, a feature observed in patients with COVID-19.

In their report, the investigators conclude that hACE2 mice, which are susceptible to SARS-CoV-2, provide a valuable small animal model for studying SARS-CoV-2 pathogenesis and for testing vaccines and therapeutics, but they also recognize that in the absence of clinical signs such as respiratory disease, other animal models that recapitulate more severe forms of COVID-19 might be needed.

Other labs are using hACE2 transgenic mice that had previously been developed for SARS research to study SARS-CoV-2 infection. Bao et al. used transgenic hACE2 mice generated by insertion of a DNA construct containing the human ACE2 coding sequence placed downstream of the mouse Ace 2 promoter in the genome of ICR mice (ACE2-hACE2). Jiang et al. used ACE2 tissue-specific transgenic mice generated by insertion of a cassette containing the human ACE2 protein under the control of a lung ciliated epithelial cell-specific HFH4/FOXJ1 promoter in
C3B6 mice (HFH4-hACE2), which had been developed in Ralph Baric's lab at the University of North Carolina at Chapel Hill.

In both studies, mice were challenged with SARS-CoV-2, monitored for clinical signs and examined for viral replication and pathological changes. Bao et al. inoculated male and female (6-11-month old) WT (control) and ACE2-hACE2 mice intranasally with SARS-CoV-2. The mice showed no clinical signs but ACE2-hACE2 mice lost weight. Viral loads were detectable in the lungs of ACE2-hACE2 animals but not in those of WT mice. ACE2-hACE2 mice developed interstitial pneumonia characterized by thickened alveolar septa accompanied with infiltration of inflammatory cells, and accumulation of inflammatory cells in alveolar cavities.

Jiang et al. inoculated male and female (8-10-week old) HFH4-hACE2 mice intranasally with the virus or vehicle (control). Several HFH4-hACE2 mice infected with SARS-CoV-2 lost weight and developed signs of respiratory distress and neurological symptoms. Viral RNA was found in the lungs, as well as in the eyes and hearts of some infected mice at lower levels, and in the brains of deceased mice. Pathological changes were observed in the lungs of infected HFH4-hACE2 mice as early as $1 \mathrm{dpi}$, which progressed into either mild or severe pneumonia after $5 \mathrm{dpi}$.

The mouse models of SARS-CoV-2 infection described in the studies differ in the tissue distribution of human ACE2 and of viral replication, in the extent of neuroinvasion, and in the severity of the disease observed. The technique used to generate the mouse, the strain, sex and age of the animals are important factors that might have influenced the phenotype of the mice. Other strains or models, which had been previously tested with SARS-CoV, should also be verified with SARS-CoV-2.

\section{Alexandra Le Bras}

Published online: 15 June 2020

https://doi.org/10.1038/s41684-020-0582-5 